\title{
The Effect of Intratesticular Injection of Human Adipose-Derived Stem Cell (hADSC) on Testicular Oxidative Stress and Spermatogenesis Process in the Varicocele Rat Model
}

Safendra Siregar ( $\sim$ safendra.sir@yahoo.co.id)

General Hospital Hasan Sadikin Bandung

Bambang Sasongko Noegroho

General Hospital Hasan Sadikin Bandung

Ricky Adriansjah

General Hospital Hasan Sadikin Bandung

Akhmad Mustafa

General Hospital Hasan Sadikin Bandung

Ananta Bonar

General Hospital Hasan Sadikin Bandung

\section{Research Article}

Keywords: human adipose-derived stem cell, varicocele, spermatogenesis, infertility, malondialdehyde, MDA

Posted Date: May 21st, 2021

DOI: https://doi.org/10.21203/rs.3.rs-492675/v1

License: (c) (i) This work is licensed under a Creative Commons Attribution 4.0 International License.

Read Full License 


\section{Abstract}

Introduction: Varicocele is the predominant cause of male infertility and was found in $19 \%-41 \%$ of men with primary infertility and $45 \%-81 \%$ of men with secondary infertility. Human adipose Derived Stem Cells (hADSC) can suppress oxidative stress in some oxidative injury model. Therefore, this study would like to investigate the effect of intratesticular hADSC injection on MDA level and spermatogenesis process by histopathological examination in the varicocele rat model.

Method: This is an experimental study. A total sampling of 9 male Wistar rats were divided into three groups. Group I consist of 1 Wistar rats without any treatment or model (sham group), group II consist of 4 Wistar rats with varicocele model without hADSC therapy (control group), and group III consist of 4 Wistar rats with varicocele model and were given injections of $1.0 \times 10^{6} \mathrm{hADSC}$ cells intratesticularly 30 days after model was made (therapy group). Testicular tissue was harvested for evaluation.

Results: In all varicocele model rats (group II and III), the result of MDA level in therapy group (2.53 $\mathrm{mol} /$ liter) was significantly lower than the MDA level in control group (4.43 mol/liter) $(p=0.01)$. On histopathological examination, the average Johnson's Score in the therapy and control group was 9,77 and 9,18, respectively. The analysis showed Johnson's score in the intervention group was significantly higher $(p=0.018)$.

Conclusion: Intratesticular injection of hADSC can help reduce MDA levels and improve spermatogenesis process, which is damaged by varicoceles.

\section{Introduction}

Infertility is one of the fundamental problems affecting human life. ${ }^{1}$ According to the Demographic and Health survey in collaboration with WHO which was conducted from 1990 to 2004, it was found that one in every four couples in developing countries was affected by infertility. ${ }^{2}$

Male infertility is a concern and contributes to $50 \%$ of total infertility cases. ${ }^{3,4}$ Varicocele is the most common cause of male infertility worldwide. Varicocele is found in $19 \%-41 \%$ of men with primary infertility and the cause of $45 \%-81 \%$ of men with secondary infertility, contributing as the most common cause of male infertility, which can be treated surgically. Studies have shown that varicoceles' incidence has been associated with increased oxidative stress, which may lead to the overproduction of reactive oxygen species (ROS) such as superoxide anion radicals, hydrogen peroxide, singlet oxygen, and nitrite proxy radicals. ${ }^{5,6}$

Reactive oxygen species are the oxygen metabolites in the form of a collection of free radicals which react easily. Reactive oxygen species will induce the lipid peroxidation process in the plasma membrane of spermatozoa and may cause damage to spermatozoa in semen and seminiferous tubules, disrupting spermatogenesis. ${ }^{1,7,10}$ 
Oxidative stress can be assessed by measuring the Malondialdehyde (MDA), an organic compound resulting from lipid peroxidation. It can be used to monitor the degree of tissue damage from oxidative stress.

Stem cell transplantation to improve organ structure or tissue function has become a new therapeutic strategy. Stem cells are capable of regenerating and differentiate into specific types of cells.

Adipose-derived stem cells (ADSC) are one of the mesenchymal stem cells (MSC) types with the most optimal therapeutic cell. These stem cells are mesenchymal stromal cells found in the perivascular adipose tissue. A study showed that ADSC could suppress oxidative stress by increasing superperoxide dismutation that will reduce oxidative stress in azoospermia condition. ${ }^{11}$

Based on the data above, it can be assumed that the administration of ADSC can suppress oxidative stress in varicoceles and improve spermatogenesis. ADSC is considered to be one of the alternative therapies besides surgical therapy of varicocele. We aim to study the effects of ADSC administration on histopathologically assessed spermatogenesis.

\section{Materials And Methods}

This research is a randomized experimental study that used experimental animals (experimental animal study). This research was conducted after obtaining approval and recommendation from the Laboratory Ethics Committee of the Institute of Biosciences, Brawijaya University Malang and were carried out in accordance with relevant guidelines and regulations. A total of nine male Wistar rats weighing 300-350 grams were used as samples and divided into 3 groups. Group I consisted of one Wistar rat without treatment or model (sham group), Group II consisted of four Wistar rats with varicocele model without hADSC therapy (control group). Group III consisted of four Wistar rats with varicocele model and was given $1.0 \times 10^{6}$ cells intratesticular hADSC injection in $100 \mu$ l of PBS solution as deep as the tunica albuginea 30 days after the varicocele model was made (therapy group). All rats were euthanized by Isoflurane gas inhalation, and following euthanasia, left orchidectomy was performed 30 days after ADSC administration in group III (Fig. 1). Testicular tissue was harvested for histopathological examination to evaluate the spermatogenesis process using Johnson's score. This study has been accepted by the Ethics Committee with the research number 013-KEP-UB-2020.

\section{Varicocele Model of Wistar Rat}

Adult Wistar rats were put under anesthesia via an intraperitoneal route using $90 \mathrm{mg} / \mathrm{kg}$ ketamine and 4 $\mathrm{mg} / \mathrm{kg}$ xylazine. A midline laparotomy incision was performed to expose the upper left abdominal quadrant. The left kidney and associated blood vessels were then exposed by displacing the intraperitoneal organ to the right. The left kidney, left renal vein, left adrenal vein, and left spermatic vein were then visualized. Through a blunt dissection, the left renal vein was freed from the adhering fat and connective tissue. The left renal vein should be exposed in a medial position from the entry point of the left spermatic vein and left adrenal vein. After the left renal vein was freed from the surrounding tissue, a 
4- 0 silk suture was used to ligate the vein. Ligation was carried out by inserting a $0.85 \mathrm{~mm}$ metal wire in the middle and knotted four times, then the wire was removed, and the left renal vein would slightly expand according to the size of the knot. This would result in consistent partial occlusion of the left renal vein in each model (Fig. 2). The intraperitoneal organs were then returned to their original position, and the abdomen was resutured by using $2-0$ silk suture. ${ }^{1}$

\section{Human Adipose-Derived Stem Cell Isolation and Characterization}

Human adipose tissue was obtained from adult male/female fat subjected to other surgeries who had agreed to donate his/her fat for research by signing informed consent. Adipose tissue was obtained with needle biopsy or liposuction aspiration method. Adipose tissue was stored at room temperature for no more than 24 hours before use. Then, the adipose tissue was washed with phosphate-buffered saline (PBS) containing 5\% penicillin/streptomycin (P/S). Culture and cell multiplication were done by thawing 1 tube, splitting once, and changing the medium once.

Flow cytometry assay was used to validate the isolated cells using the following markers: CD105 (+), CD45 (-). In the validation application, cell viability was at $86.78 \%$, with $1 \mathrm{cc}$ each containing $1.0 \times 10^{6}$ cells for use. All tissue harvesting procedures, cell isolation, culture, and characterizations were conducted in the Tissue and Cell Bank of Dr. Soetomo General Hospital Surabaya, sample-numbered with 120.E/MSC/Penelitian/AdiposeTasijo_09/060220 and were declared suitable for the application.

\section{Johnson's Score}

One of the parameters for evaluating spermatogenesis in the seminiferous tubules is by using Johnson's score. The testicular tissue is dehydrated through a graded series of $70-100 \%$ ethanol and embedded in paraffin. One portion with a diameter of $5 \mu \mathrm{m}$ was obtained to be deparaffinated and stained with Hematoxylin-Eosin (H\&E), which was then examined under a light microscope. The histopathological grade of spermatogenesis was assessed between 1 and 10 based on the development of sperm cells found in at least 5 microscopic areas in 100 seminiferous tubules. Johnson's total score is then determined by dividing the total score by the number of tubules evaluated.

\section{Result}

\section{Left Internal Spermatic Vein Width}

The success of the varicocele model was assessed based on the left internal spermatic vein width of the male rats 4 weeks after ligation. Comparison of mean internal spermatic vein width in each group is shown in Table 1. If Group II and III were combined into 1 group, namely the group of mice with a varicocele model, and were compared to group I without varicocele model, it was shown that partial ligation of the left renal vein was proven to induce the development of artificial left varicocele in mice. The mean internal spermatic vein width in the group of rats with varicocele model was $0,70 \mathrm{~mm}$. This result was greater compared to group I (sham) without varicocele model, which was $0.23 \mathrm{~mm}$. $(p=0.005)$. 
Table 1

Comparison of Left Internal Spermatic Vein Width

\begin{tabular}{|lllll|}
\hline Group & $\mathbf{N}$ & \multicolumn{3}{l|}{ Left Internal Spermatic Vein Width (mm) } \\
\cline { 2 - 5 } & & Mean & Std Deviation & Range \\
\hline Without Varikokel & 1 & 0.23 & - & - \\
\hline With Varikokel & 8 & 0.7 & 0.070 & $0.52-0.72$ \\
\hline $\mathbf{p}=\mathbf{0 . 0 0 5}$ & & & & \\
\hline
\end{tabular}

\section{MDA Concentration}

There was a significant difference in MDA levels between group II (control) and group III (therapy) as shown in Fig. $3(p=0.01)$. The mean MDA levels in the therapy group were lower $(2.53 \mathrm{~mol} / \mathrm{liter})$ than the group without ADSC (4.43 mol/liter).

\section{Histological Examination (Johnson's Score)}

There is also a significant difference in Johnson's Score between group II (control) and group III (therapy) (Figs. 3 and 4) The average value of Johnson's Score in group III (therapy) was higher than group II (control). Group III, who received ADSC, had an average Johnson's score of 9.77, whereas group II without ADSC had an average Johnson's score of 9.18. Data analysis by using the Kolmogorov Smirnov test showed that the data distribution was not normal $(p<0.05)$. Based on the results of normalization tests that were not normally distributed, data analysis was performed using the Mann-Whitney test. There was a significant difference in the mean value of Johnson's Score between treatment groups with $p=0.018$

\section{Discussion}

Several theories hypothesized the relationship between varicocele and infertility, including increased temperature in germ cells sensitive to temperature changes, cadmium accumulation, testicular hypoxia, and metabolite reflux, leading to increased stress oxidative. This oxidative stress causes damage to the function and maturity of spermatogenesis, membrane peroxidation, and hormonal disturbances, which reduce male reproductive potential in the form of decreased sperm count, shape, and motility. ${ }^{14}$

In this study, it was found that there is a significant difference in mean MDA levels between groups $(p=$ $0.01)$. MDA levels in the therapy group were lower $(2.53 \mathrm{~mol} / \mathrm{liter})$ than the group without ADSC (4.43 $\mathrm{mol} / \mathrm{liter}$ ), this result was in line with other studies showing that ADSC can self-renew in the vascular stroma and can influence local regulation of angiogenesis and blood vessels remodeling, thereby suppressing oxidative stress by increasing the activity of superoxide dismutase which will cause a decrease in the formation of MDA. ${ }^{15-17}$ 
Varicoceles can cause progressive deterioration of the Sertoli cells and trigger the release of spermatogonia cells before their peak maturity. Histopathologic examination in varicocele may show normal and defective spermatogenesis. ${ }^{18}$

In a previous study conducted by Qin et.al, it was found that varicocele can cause damage to the endothelial part of the testis and seminiferous tubules, as well as interfering with the spermatogenesis process. They conducted a study by ligating the left renal vein in mice until it was enlarged by two or more times and found a significant reduction in sperm count compared to the control group. ${ }^{19}$

This study showed that the group of mice with varicocele receiving ADSC therapy had a better Johnson's score $(9,77)$ than the group of mice with varicocele without receiving therapy $(9,18)(p=0.018)$.

The group model of mice with varicocele that received ADSC therapy had an average Johnson's Score of 9.77, with the highest score of 9,93 and the lowest score of 9,4. This score showed normal spermatogenesis histopathologically. Normal spermatogenesis is characterized by the presence of seminiferous tubules that are covered by a thin basal membrane and germinal epithelium, indicating normal sperm development from spermatogonia to spermatocytes, including spermatids and spermatozoa.

Whereas in the group model of varicocele mice without ADSC therapy, the lowest Johnson's score was 8.33. The picture in this sample shows the histopathological features of hypospermatogenesis, where the germinal epithelium shows a normal stage of sperm development, but the number is greatly reduced.

We used human ADSC in this study because the number of fat cells from liposuction was abundant, and in the future, we aim to use human ADSC in human patients. Therefore we started using human ADSC in mice. Human ADSC will not induce xenogenic immunological reactions because it does not express $\mathrm{MHC}$ class II.

We performed ADSC intratesticular injection method and found a significant result of spermatogenesis improvement. Intratesticular injection of ADSC gives a better result compared to intravenous injection because there is a blood-testis barrier in the intravenous line that may prevent stem cells from entering testis tissue through the circulation. In addition, it will prevent ADSC from getting trapped in the lungs due to their large size of molecules. ${ }^{20}$ Another advantage of intratesticular administration of stem cells is that they can significantly protect from testicular damage. Although complications from injection procedure may cause anti-sperm antibody formation that can lead to infertility, long term follow up is necessary. ${ }^{21}$

Sampling was carried out on the 30th day after ADSC administration to the group of rats with varicocele. The 30 days assessment for sampling was used to assess the success of the effect of ADSC on the spermatogenesis cycle of Wistar rats, ranging from 30 to 48 days. $^{22}$

Stem cell injection may prevent tissue damage caused by oxidative stress. After administration of stem cells, stem cells were integrated into interstitial area and seminiferous tubules and into the vascular walls 
of the testis, secreting various growth factors, such as Basic Fibroblast Growth Factor and Stem Cell Factor, which would reduce the level of oxidative stress, regulating hormonal levels and promoting regeneration of Leydig cells and germ cells, which would improve the process of spermatogenesis and increase Johnson's Score in the testis. ${ }^{21}$ Intratesticular injection of ADSC also has the effect of increasing and repairing testosterone levels. It suggests that ADSC may improve the endocrine function of the testis. $^{20}$

Male infertility could be a consequence of interference in germ cells, Sertoli or Leydig due to varicocele. This can be corrected through stem cell transplantation.

\section{Conclusion}

Based on the results and discussion of this study, it can be concluded that the administration of ADSC may improve the spermatogenesis process damaged by varicoceles. This shows the benefits of ADSC as a stem cell therapy which may be further investigated as one of the therapeutic options in improving the spermatogenesis process for patients with varicocele.

\section{Declarations}

\section{Ethics approval and consent to participate}

We have read and complied with the policy of the journal on ethical consent and the standards of animal care, as stated in the Guide to Authors. The work has been approved by our institution's Institutional Review Board with number 013-KEP-UB-2020

\section{Consent for publication}

Not Applicable

\section{Availability of data and materials}

All data generated or analyzed during this study can be requested to the corresponding author.

\section{Competing interests}

None

\section{Funding}

This study was supported from internal Universitas Padjadjaran grant

\section{Authors' contributions}

Conception and design: All authors 
Administrative support: All authors

Provision of study materials or patients: All authors

Collection and assembly of data: All authors

Data analysis and interpretation: All authors

Manuscript writing: All authors

Final approval of manuscript: All authors

\section{Acknowledgements}

The authors have no acknowledgments.

\section{References}

1. Noegroho BS. Penilaian Stres Oksidatif Varikokel Kiri Buatan Pada Kelinci (Oryctalagus cuniculus) Model Yang Ditangani Dengan Atau Tanpa Antioksidan Vitamin C. Bandung: Universitas Padjadjaran; 2010.

2. Mascarenhas MN, Flaxman SR, Boerma T, Vanderpoel S, Stevens GA. National, Regional, and Global Trends in Infertility Prevalence Since 1990: A Systematic Analysis of 277 Health Surveys. PLOS Medicine. 2012;9(12).

3. Agarwal A, Mulgund A, Hamada, Chyatte MRA. Unique view on male infertility around the globe. Reprod Biol Endocrinol 2015;26:37.

4. Wong WY, Merkus HM, Thomas CM, SteegersTheunissen RMGAZRP. Effects of folic acid and zinc sulfate on male factor subfertility: a double-blind, randomized, placebo-controlled trial. Fertil Steril. 2002;77(3):491-8.

5. Agarwal A, Sharma R, Harlev A, Esteves SC. Effect of varicocele on semen characteristics according to the new 2010 World Health Organization criteria: a systematic review and meta-analysis. Asian J Androl. 2016;18(2):163.

6. Khatri DK, Juvekar A. Preliminary phytochemical and antioxidant evaluation of a polyherbal formulation. Int J Phytopharm. 2013;4(5):322-8.

7. Saleh RA, Esfandiari N, Sharma RK. Diagnostic and prognostic value of measurement of reactive oxygen species in neat semen. American Society for Reproductive Medicine. 2001.

8. Smith, R., Kaune, H., Parodi, D., Madariaga, M., Ríos, R., Morales, I., 2006. Increased sperm DNA damage in patients with varicocele: relationship with seminal oxidative stress. Hum. Reprod. 21 (4), 986-993.

9. Bromfield, E.G., Anderson, A.L., McLaughlin, E.A., Nixon, B., 2015. The impact of oxidative stress on chaperone-mediated human sperm-egg interaction. Hum. Reprod. 30 (11), 2597-2613. 
10. Di Meo, S., Reed, T.T., Venditti, P., Victor, V.M., 2016. Role of ROS and RNS sources in physiological and pathological conditions. Oxid. Med. Cell. Longev.

11. Cakici C, Buyrukcu B, Duruksu G, Haliloglu AH, Aksoy A, Isık A. Recovery of fertility in azoospermia rats after injection of adipose-tissue derived mesenchymal stem cells: the sperm generation. Bio Med Res Int. 2013.

12. Katz MJ, Najari BB, Li PS, Goldstein M. The role of animal models in the study of varicocele. Translational Andrology and Urology. 2014;3(1):59.

13. Dohle GR, Elzanaty S, van Casteren NJ. Testicular biopsy: clinical practice and interpretation. Asian Journal of Andrology. 2011;14(1), 88-93.

14. Su JS, Farber NJ, Vij SC. Pathophysiology and treatment options of varicocele: An overview. Andrologia. 2020

15. Asadi, Nematollah, et al. Effect of royal jelly on testicular antioxidant enzymes activity, MDA level and spermatogenesis in rat experimental Varicocele model. Tissue and Cell.2019.57(1):70-77.

16. Sun, Ge, et al.Effects of adipose-derived stem cells on antioxidant enzymes and malondialdehyde in radiation damaged rat. Radiation Protection Bulletin.2012.32(4): 1-5.

17. Ge, Yansong, et al.Adipose-derived stem cells reduce liver oxidative stress and autophagy induced by ischemia-reperfusion and hepatectomy injury in swine." Life sciences.2018.2(1):62-69.

18. Hamada A, Esteves SC, Agarwal A. Varicocele and male infertility: current concepts, controversies and consensus. Springer; 2015.

19. Qin Q et al. Aberrant expressions of stem cell factor/c-KIT in rat testis with varicocele. Journal of the Formosan Medical Association. 2016

20. Meligy FY, Elgheed AT, Alghareeb SM, Therapeutic effect of adipose-derived mesenchymal stem cells on Cisplatin induced testicular damage in adult male albino rat. Ultrastructural Pathology. 2019

21. Zhou LH et al. Protective Effects of Uncultured Adipose-Derived Stromal Vascular Fraction on Testicular Injury Induced by Torsion-Detorsion in Rats. Stem Cells Translational Medicine. 2018

22. Hikim AP, Maiti BR, Ghosh A. Spermatogenesis in the Bandicoot Rat. I. Duration of the Cycle of the Seminiferous Epithelium. Archives of Andrology.2009

\section{Table}

Table 1 Comparison of Left Internal Spermatic Vein Width

\begin{tabular}{ccccc}
\hline & & \multicolumn{3}{c}{ Left Internal Spermatic Vein Width $(\mathrm{mm})$} \\
\cline { 3 - 5 } Group & $\mathrm{N}$ & Mean & Std Deviation & Range \\
\hline Without Varikokel & 1 & 0.23 & - & - \\
With Varikokel & 8 & 0.7 & 0.070 & $0.52-0.72$ \\
\hline & & & & \\
$\mathbf{p}=\mathbf{0 . 0 0 5}$ & & & &
\end{tabular}


Figures
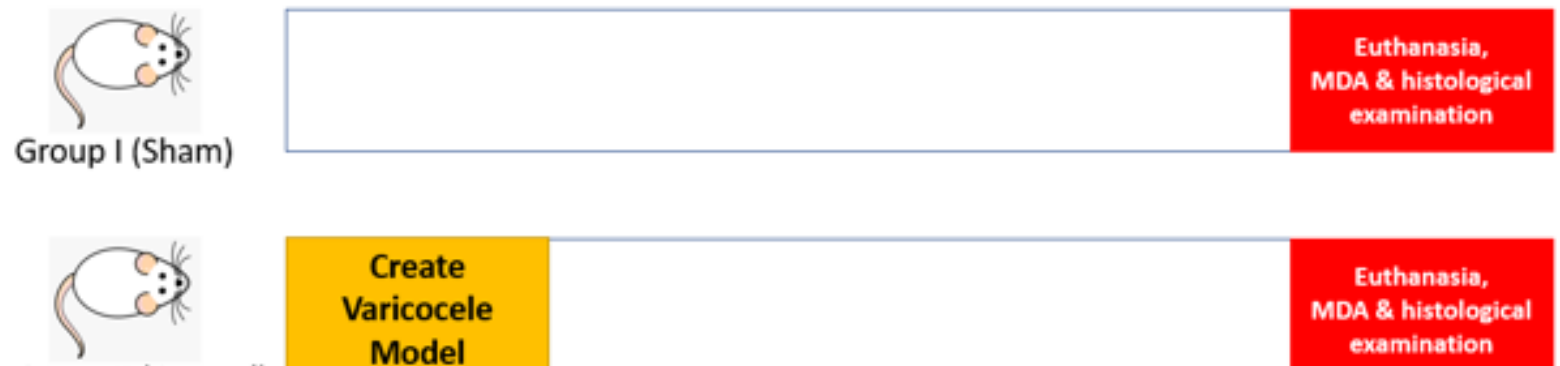

Create
Varicocele
Model

Euthanasia,

MDA \& histological

Group II (Control)

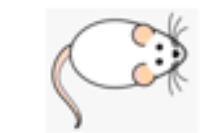

Group III (Therapy)

\begin{tabular}{|c|c|}
\hline Create & hADSC \\
Varicocele & Injection \\
Model &
\end{tabular}

Euthanasia,

MDA \& histological

examination

Day $1^{\text {st }}$

Day $30^{\text {th }}$

Day $60^{\text {th }}$

\section{Figure 1}

Timeline in procedure and group control of animal model

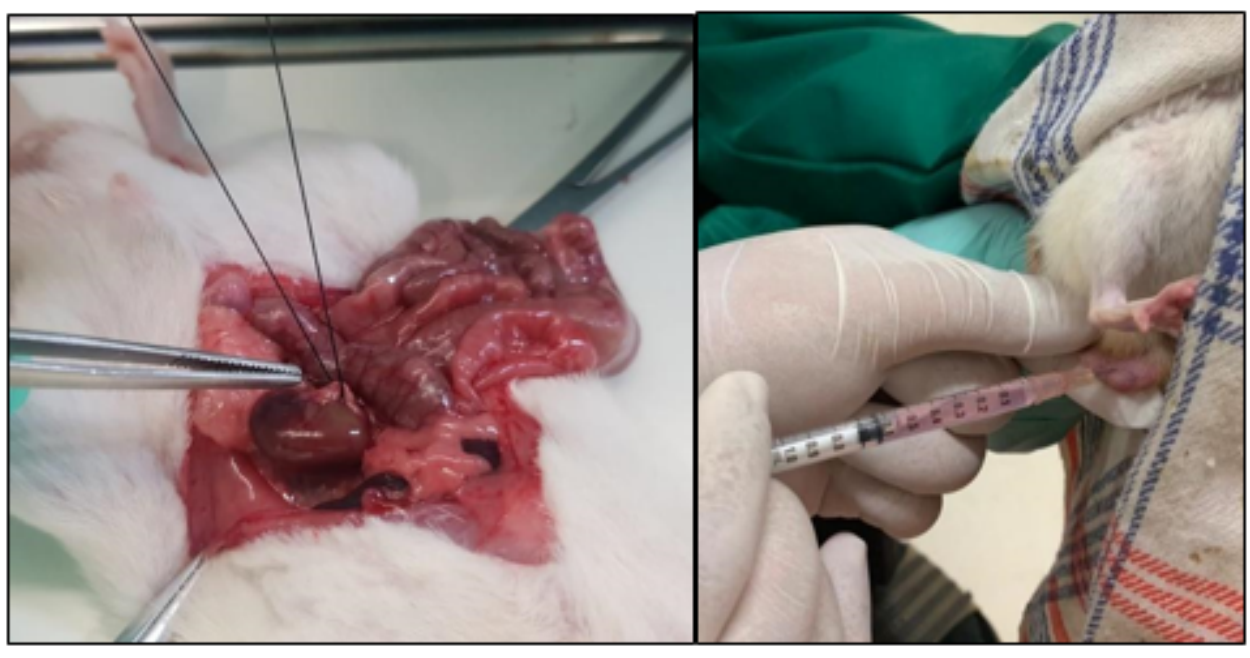

\section{Figure 2}

internal spermatic vein ligation and Intratesticular ADSC injection 

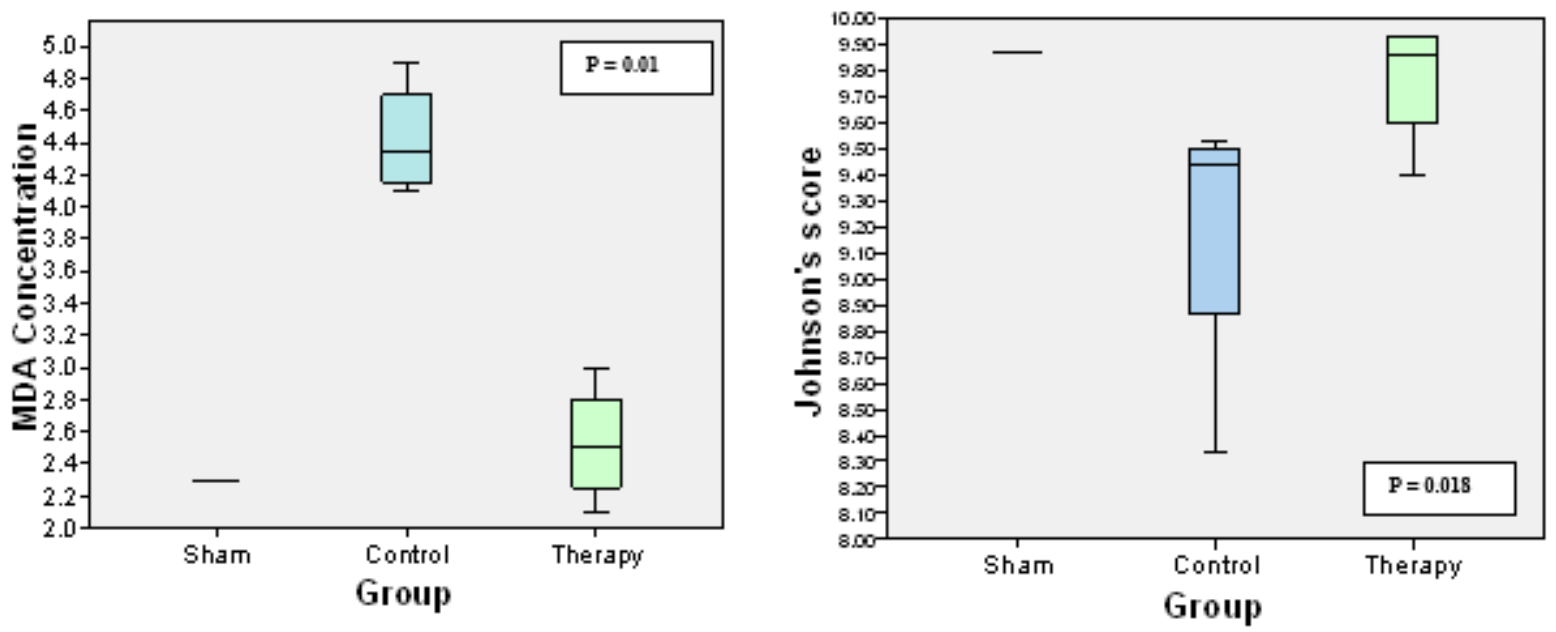

\section{Figure 3}

Result of johnson's score and MDA concentration between groups

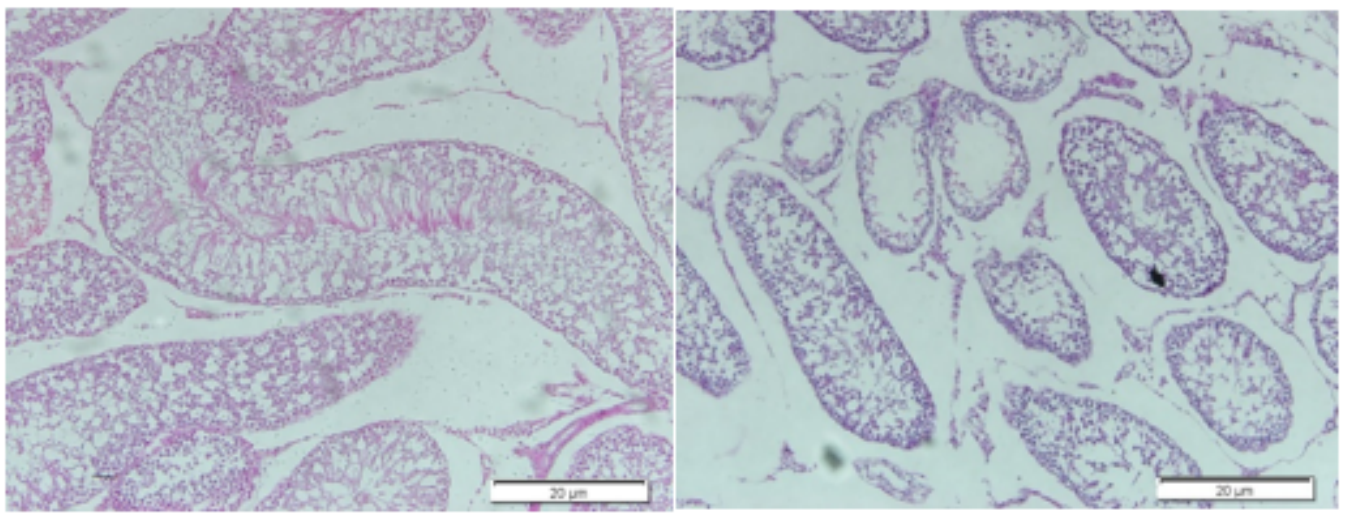

Figure 4

A) Normal Spermatogenensis H\&E x100. B) Hypospermatogenesis H\&E x100 